\title{
UPGRADING MOOC STUDENTS' ENGAGEMENT AND PARTICIPATION IN HUMANITIES-ORIENTED ONLINE COURSES: THE EXAMPLE OF THE MOOC BASED ON THE PROJECT "DETECT"
}

\author{
Jan Baetens, Roberta Pireddu, \& Frederik Truyen \\ Literary Theory and Cultural Studies, KU Leuven (Belgium)
}

\begin{abstract}
Massive Online Open Courses (MOOCs) have become a grounded reality and a stable concept in the distance education panorama with worldwide universities continuously creating and offering every year broad selections of online courses. Nevertheless, despite the many developments in terms of individual and distance learning approaches, it is indetermined if MOOCs can deliver effective pedagogical methods and tools suitable for the implementation of online courses in the categories of art and humanities as well as in creating environments that give equal space to the two complementary layers of distance learning and distant teaching. Consequently, also the development of a valid, and captivating e-learning experience able to effectively reach out to students of different backgrounds, creating an impactful learning community represents a challenge. This issue acquires certain relevance particularly in relation to the much-debated question around the most effective pedagogical methodology to deliver humanities-oriented knowledge in a distant learning context. This paper provides an overview of the educational and pedagogical formulas adopted for the creation of a MOOC on European Crime Fiction, currently being developed in the framework of DETECt - Detecting Transcultural Identity in European Popular Crime Narratives (https://www.detect-project.eu/) a project funded by European's Union Horizon 2020. The MOOC concept presented in the framework of this research concentrates on the development of mixed e-learning and e-teaching strategies, that leverages the application of pedagogical elements like social network and independent learning and combines them with users' engagement methods. On the one hand, this research aims to challenge the debate related to the effectiveness of teaching and learning a humanities-oriented subject in a distance learning environment. On the other hand, intends to recreate a vibrant learning community capable of broadening the academic research carried out by the project enabling the collaboration between the MOOC public and the researchers and teachers.
\end{abstract}

Keywords: E-learning, MOOC, learning community, online learning, e-teaching.

\section{Introduction}

This paper explores the instructional and pedagogical strategies utilized to create and deliver a MOOC on European Crime Fiction developed within the framework of DETECt - "Detecting Transcultural Identity in European Popular Crime Narratives" (https://www.detect-project.eu/), a large collaborative project funded by European Union's Horizon 2020.

Over the last decade, MOOCs development and diffusion lead to a considerable improvement in the instructional methodologies and approaches used to enhance the distance learning experience, partially encouraging the overcome of the issue of the suitability of humanities subjects for online learning environments (Reichard, 2013). A consequence of this change of tendency can be recognized in the slight increase in the percentage of humanities MOOCs offered by Higher Educational Institutions (Shah, 2020). However, despite these recent educational and technological upgrowths the creation of humanities-oriented MOOCs still represents a challenge that involves not only the learning but also the teaching context (Limarev et al, 2020). The essential role played by e-teaching both in the instructional development of the distance learning ecosystem and in the creation of a learning network appears in fact still underrated and in need of gaining more attention by the leading MOOCs platforms. While elements such as scalability, user capacity, and distance learning methods were further developed, teachers and educators' functions received marginal attention and were left behind. (Blackmon, 2018). Besides, in this context also the question around the right educational methodology to deliver humanities online courses, that support students' interaction in a massive environment, is a debated issue (Kaplan \& Bornet, 2014). 
This research aims to design a learning environment based on a humanities subject where equal attention is given not only to distance learning and to the enhancement of the student's engagement but also to the distance teaching context and methods. This investigation also intends to fit into the still intense discussion revolving around the most impactful methodologies for delivering and teaching humanities-oriented MOOCs.

\section{The DETECt Project}

Funded by the European Union Horizon 2020 Research \& Innovation Program, DETECt
DETECt - Detecting Transcultural Identity in European Popular Crime Narratives (https://www.detect-project.eu/) is a combined initiative involving researchers and professionals from eighteen different European institutions. DETECt aims to investigate the contribution of European crime narratives in influencing the creation and representation of a European cultural identity. Through a broad series of cultural and educational initiatives, the project's intention is to bring the public's attention to the DETECt research outcomes fostering the dialogue between crime fiction enthusiasts and academia.

One of the vehicles that intend to lead to the dissemination of the research results and to the expansion of the DETECt learning community is represented by the creation of a MOOC on "Euro Noir: Transcultural Identities in European Popular Crime Narratives" (https://www.edx.org/course/euro-noirtranscultural-identities-in-european-popular-crime-narratives), designed and developed by six of the partner's institutions that collaborate to the project and that comprehend KU Leuven -which as member of the edX consortium manages the development process of the MOOC-, University of Bologna, University of Limoges, University of Debrecen, University of Bucharest, Aalborg University. The MOOC is based on two different educational purposes. On the one hand, it intends to create an environment where not only student interaction but also teachers' communication, especially during the development of the distance teaching approach, are emphasized. On the other hand, it aims to leverage the DETECt knowledge network in order to create a vibrant learning community: a purpose in line with the DETECt intention of promoting cultural diversity and intercultural dialogue around the topic of European Crime Fiction.

\section{Design and educational challenges of a MOOC on European crime fiction}

Although the educational and technological improvement in the online learning field allowed the acquisition of more sophisticated pedagogical methodology for the MOOCs' development, some elements that distinguish the MOOCs concept are still being considered the primary obstacles in the creation of valid humanities-oriented MOOCs (Kaplan \& Bornet, 2014 and Blackmon 2018). One of the basic characteristics of MOOCs, the "massive" component, is recurrently indicated as one of the most debatable due to the fact that the presence of a very broad and diverse audience represents a challenge for developing a MOOC in a sector such as the humanities that usually bases its classes on a low number of students (Blackmon, 2018). In this context, in fact, the "scale" element could affect the co-construction of knowledge hindering the students' interaction not only with the other students but also with the teachers (Blackmon, 2018). Also, it represents a challenge in relation to the student's assessment, which in learning environments based on a humanities subject cannot uniquely rely on an automatic grading system as normally can be observed in science MOOCs (Kaplan \& Bornet, 2014).

These challenges are further emphasized by the internal structure and pedagogical approach of some of the MOOCs leading platforms and emerged during the implementation of the MOOC based on the DETECt research project, hosted on the edX platform. EdX usually orients their courses on the xMOOCs model -based on the behaviorist theory of learning-. These are generally built using a static textbook structure that encourages independent learning but that also tends to hinder student interaction. In fact, the only interaction medium, which is represented by discussion forums, does not completely allow a fluid synergy among the participants since this component is presented more as a tool for asking questions than as a medium to boost users' discussion (Waller et al, 2019). Besides, as many other platforms, also edX does not offer equal space to the two equally essential layers of distance learning and distant teaching and does not guarantee support to the educators during the process of the content design. These elements represented the main challenges to the development of a MOOC whose main objectives were not only to encourage the creation of a learning community within the online course itself but also to build an environment that could support teachers in the creation of a coherent educational framework, essential for the knowledge delivery.

In order to overcome these issues, we adapted the design of the MOOC to our main educational purposes, operating on the two levels of distance learning and distance teaching. On the one hand, through a thorough design of the learning activities, we aimed to point to the need of different types of 
students by combining independent learning approaches with forms of social networks. This is intended to encourage students to go beyond the platform textbook structure and expand and apply their knowledge by navigating outside the platform. On the other hand, we integrated on the same edX platform elements belonging both to the $\mathrm{xMOOC}$ and the cMOOC models. This enabled the creation of a more flexible educational methodology that could also involve elements of participatory teaching.

\subsection{Distance teaching strategy}

In this context, one of the major elements of concern during the development of distance education environments is represented by the design phase of the learning structure, which implies teachers' and educators' commitments and collaboration in the creation of an impactful virtual environment (Markova et al 2017). In this context, students' satisfaction is in fact directly influenced by the educational structure of the distance learning environment as well as by educators' ability to manage knowledge delivery and interaction among the participants (Markova et al 2017). For this reason, teachers play a crucial role in the delivery of an engaging and coherent educational structure (El Emrani et al 2017). Consequently, it is necessary not only that they develop further expertise on adapting teaching methods to distance education environments but also that their role becomes fully recognized as essential in the delivery of a MOOC (Markova et al 2017). Distance learning and distance teaching should not only coexist and be equal elements of the same structure but their mutual communication should be allowed and maintained.

Teacher's training and teacher's communication represented the main challenge for the development of the online course based on the DETECt project since the edX platform used for the development of this MOOC does not offer valid support for the development of the aforementioned elements. In order to address this issue and enable both a smooth collaboration among the teachers and instructional design skills acquisition we have combined two different components:

1) A Moodle instance linked to the main DETECt web portal (https://www.detectproject.eu/portal/learning/) and utilized during the MOOC instructional design process as a content repository for the unique use of the teachers involved in the MOOC development. The platform was in fact used to gather the learning material and test it before it was included in the online course. The use of Moodle helped develop consistency in the content creation allowing teachers' remote communication and collaboration.

2) The e-teaching training was supported from the beginning of the designing process through the adoption of KU Leuven pedagogical and technical support. This resulted crucial in providing the teachers with further skills related to the creation and shaped of the environment in line with the learning goals of the project.

\subsection{Distance learning strategy}

Since the diffusion of the MOOCs concept, students' interaction and involvement within the distance learning environment constitutes the central element of many research studies (Markova et al, 2016). Some traditional characteristics of the e-learning contexts - such as the educational effectiveness of independent learning, the MOOCs' scalability, and learners' background diversity - have often been objects of critics and labeled as elements that would hinder knowledge acquisition. Although at the same time the benefits of distance learning cannot be denied -especially in extreme contexts such as during the COVID19 pandemic -, the debate around teaching and learning humanities subjects in a distance learning environment is still debated (Limarev et al, 2020).

Exploiting the many potentialities of a distance learning environment in order to create not only a coherent and effective learning experience but also a solid learning community was at the base of the development process that leads to the creation of the MOOC on "European Crime Fiction". From a distance learning point of view, the main goal was to challenge the main structure of the edX course based on the independent learning approach and to apply strategies of user engagement and social networking in order to enhance students' interaction and participation.

The MOOC based on the DETECt research project was designed as an environment where autonomous learning and interactive and participatory learning were equally balanced. In this regard the following elements were at the base of the DETECt MOOC's structure and approach:

- The use of a series of external ecosystems developed within the DETECt project, which comprehends: The DETECt Portal, a platform enriched with additional learning material, and the DETECt Atlas, a platform that allows students to explore European crime fiction using and playing with interactive maps.

- The use of interactive collaborative environments (e.g., Wakelet, MindMap) to allow social interaction and collaboration among the students.

On the one hand, the acquisition of these components and the parallel use of the edX platform's structure would enable students who prefer undertaking a learning path centered on individual studying to 
still acquire the right knowledge and skills for understanding and discussing European crime fiction. On the other hand, the presence of collaborative tasks, which include not only group discussion within the platform but also the interaction with further learning material outside the platform, would encourage students to go beyond the traditional independent learning trajectory.

\section{Conclusion}

The pedagogical and instructional approaches described in this paper and utilized for the development of a humanities-oriented MOOC on "European Crime Fiction" aimed to challenge the traditional MOOC structure highlighting not only the complementary role of distance learning and distance teaching within the same environment but also the possible interrelation between efficient e-teaching and students' satisfaction. The methodologies and strategies applied for the creation of this online environment represent the first step of this research study. This will be followed by a thorough investigation both of student's engagement and behavior during their learning process as well as the efficacy and consistency of the two parallel ecosystems dedicated on the one hand to distance learning and on the other hand to distance teaching with regards to the knowledge delivery and management.

\section{Acknowledgments}

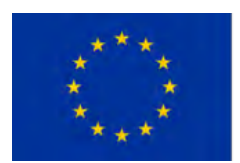

This project has received funding from the European Union's Horizon 2020 research and innovation programme under grant agreement No. 770151

The views and opinions expressed in this publication are the sole responsibility of the author(s) and do not necessarily reflect the views of the European Commission.

\section{References}

Blackmon, S., (2018). MOOC Makers: Professors' Experiences with Developing and Delivering MOOCs. International Review of Research in Open and Distributed Learning, Volume 19(4), 75-88.

DETECt, Detecting Transcultural Identity in European Crime Narratives. (2018-2021) Retrieved May $14^{\text {th }}, 2021$ from https://www.detect-project.eu/

El Emrani, S., El Merzouqi, A., Khaldi, M. (2017). The MOOC: Challenges and Opportunities from a Pedagogical View. International Journal of Computer Applications, Volume 162(9), 25-29.

Kaplan, F., Bornet, C. (2014). A PREPARATORY ANALYSIS OF PEER-GRADING FOR A DIGITAL HUMANITIES MOOC. Digital Humanities 2014: Book of Abstract. Retrieved May 14 ${ }^{\text {th }}, 2021$, from: https://infoscience.epfl.ch/record/200911? ln=en.

Limarev, P. V., Limareva, Y. A., Zinovyeva, E. G., Vikulina, V. V., Votchel, L. M., Grygoryants, I. A. (2020). Distance learning online technologies as a promising form of the educational process for the humanities. Journal of Physics: Conference Series, Volume 1691. Retrieved on 12th May, 2020, from: https://iopscience.iop.org/article/10.1088/1742-6596/1691/1/012188.

Markova, T., Glazkova, I., Zabarova, E. (2017). Quality Issues of Online Distance Learning. Procedia Social and Behavioral Sciences, Volume 237, 695-691.

Reichard, C., (June 4th, 2013). MOOCs face challenges in teaching humanities. Retrieved May 5th, 2021 from https:/www.stanforddaily.com/2013/06/04/moocs-face-challenges-in-teaching-humanities/.

Shah, D., (November 30th, 2020). By The Numbers: MOOCs in 2020. Retrieved May 11th, 2021, from https://www.classcentral.com/report/mooc-stats-2020/.

Waller, D. R., Douglas, K. A., Nanda, G. (2029). A Case Study of Discussion Forums in Two Programming MOOCs on Different Platforms. American Society for Engineering Education. Retrieved on 12th May, 2020, from: https://peer.asee.org/a-case-study-of-discussion-forums-intwo-programming-moocs-on-different-platforms.pdf. 